\title{
User-driven Design of an Ontology-based Ambient-aware Continuous Care Platform
}

\author{
Femke Ongenae, Ann Ackaert, Filip De Turck \\ INTEC Broadband Communications Networks research \\ Group (IBCN) \\ Ghent University (UGENT) - IBBT \\ Gaston Crommenlaan 8, bus 201, 9050 Ghent, Belgium \\ Femke.Ongenae@intec.ugent.be
}

\author{
Piet Verhoeve \\ Televic N.V. \\ Leo Bekaertlaan 1, 8870 Izegem, Belgium \\ p.verhoeve@televic.com
}

\author{
An Jacobs, Annelies Veys \\ Research Centre for Studies on Media, Information and \\ Telecommunication (SMIT) \\ Brussels University (VUB) - IBBT \\ Pleinlaan2, 1050 Brussels, Belgium \\ An.jacobs@vub.ac.be
}

\author{
Mieke Van Gils \\ Centre for User Experience Research (CUO) \\ K.U. Leuven - IBBT \\ Parkstraat 45, bus 3605, 3000 Leuven, Belgium \\ Mieke.vangils@soc.kuleuven.be
}

\begin{abstract}
The ultimate ambient intelligent care room environment would be able to sense the needs and preferences of the patients and nurses and adapt itself accordingly. This implies an emerging demand for the integration and exploitation of heterogeneous information available from different technologies. Nowadays, the nurse is responsible for orchestrating all these technologies, which slows down the adoption rate. In this paper, a context-aware, ambient aware and pervasive framework is proposed that tackles this integration problem by using an ontology. Rules, defined on top of this ontology, implement algorithms to optimize and automate care tasks. To increase the acceptance of the new technology, a user-driven development process is used which involves the stakeholders in every step of the design of the ontology and algorithms. To make the framework adaptable to future needs, a self-learning component is introduced that detects trends in the execution of the Rules and adapts the system accordingly.
\end{abstract}

User-driven, Continuous care, Ontology, Ambient intelligent, Self-learning

\section{INTRODUCTION}

In recent years the complexity in nursing organizations has been increasing due to societal factors such as the increase of the care unit size, the increase of specialized care and the lack of nurse staffing which requires a more efficient use of resources. In addition to these inherent factors, a further increase of complexity is due to the high amount of technology that is being introduced for the staff (e.g. medical equipment, pagers, alert redirecting and electronic medical records) as well as for the environment (e.g. building automation for energy control and comfort functions for the patient). In future years these complexity trends will continue due to upcoming technologies, such as location aware services, and computerized decision support systems, and an aging society, which translates into an increasing need for care and a decreasing number of available staffing.
However, based on current state of the art systems the adoption rate of further innovative techniques is decreasing and will come to a complete stop due to the fact that the users are directly faced with the complex technologies. Indeed, today each technology is being equipped with intelligence on its own, however it is the user (nurse) that is orchestrating the technologies and moving information from one technology to another. This is a time-consuming job [1]. As an example, suppose a patient that had an eye operation and needs to be in a dark/dim environment to heal. Today, it is the nurse that has to configure the room to dim the lights each time he/she enters. If the nurse presses the wrong button, he/she causes pain for the patient. However, if the system would be aware of the patients and nurses needs, namely darkness for the patient and sufficient light for the nurse to work, the system can automatically put the light to the correct level when it detects that the nurse enters the room.

The ultimate ambient intelligent care room environment would be able to sense the needs and preferences of all the actors involved, taking into account the pitfalls of a too static and user depowering system, which gives the users very limited control. Ambient intelligence, pervasive/ubiquitous computing and context-aware techniques could be used to implement such an intelligent care room [2]. The common denominator of these techniques is that the technology will blend into the background of the environment, user interfaces will be integrated into other attributes, such as furniture, and sensors and actuators will be able to sense and adapt our environment [3]. This implies an emerging demand for the integration and exploitation of the heterogeneous information available from all these sources. It is clear however that the introduction of context awareness into real products and services is lagging behind what could be expected. Criel and Claeys [4] state that this is due to inadequate techniques for real personalization of the services and tackling problems such as the fear for technology and the need for control. Technology and environment should melt into each other, making for an 
interaction that should go beyond traditional notions of usefulness and usability, and focus on the soft aspects of interaction [5] such as automated and personalized alerts.

In this paper, a context-aware, ambient intelligent and pervasive framework is proposed that tackles these problems by employing an ontology. Ontologies [6] are used to model knowledge about a certain domain in a formal manner. This common, agreed data-format can then be used to integrate and exchange data from different sources which enables more integration, re-use and collaboration. Simple business logic of an application, such as classification Rules [7], can be modeled in an ontology, while more complex logic can be expressed by defining Rules [8] on top of this ontology. This allows defining algorithms that take advantage of the information in the ontology, which models the current context, to automate, optimize and personalize the continuous care of patients. Referring back to the previous example, this means that the ontology could model the condition of the patient (sensitive to light) and the location of the nurse (in the room). Algorithms can be defined that automatically put the light to the correct level when it detects that the nurse enters the room namely dim lights for a patient with light sensitivity and bright lights for other patients. Afterwards, the nurse can decide to overrule this Rule by adjusting the light level in the room manually.

The incorporation of ontology engineering tasks in knowledgeempowered organizations such as hospitals can prove to be a hindrance if not done in a way that is seamless to the day-today activities of the nurses, patients and doctors [9]. To resolve this issue, the construction of the ontology and the accompanying algorithms of the proposed framework are userdriven by including the stakeholders in every step of the design and development process. This will not only facilitate the integration and acceptance of this new technology and take away their fear of the technology, but it will also empower the staff members to manage the ontologies, make suggestions for changes and thus shape their common information space to their needs. To make the framework even more adaptable to future needs, a self-learning component is introduced that detects trends in the execution of the Rules based on the information in the ontology and adapts the system accordingly.

The remainder of this paper is organized as follows. Section II details the architecture of the proposed framework. The userdriven approach of developing the ontology and algorithms is discussed in Sections III and IV. Section V details the selflearning component. Section VI contains the conclusions.

\section{GENERAL ARCHITECTURE}

The general architecture of the proposed context-aware, ambient-intelligent framework to support continuous care of patients is visualized in Fig. 1. The Knowledge Management Component is contains the Ontology which models all the necessary context information about the hospital such as the profiles of the staff and information about the patients and the environment. It also contains all the data that conforms to this Ontology. An OWL Reasoner is used to check the consistency of the model and to infer new knowledge out of the data available in the Ontology. The layer also holds all the Rules that work with the information in the ontology. Rules are used

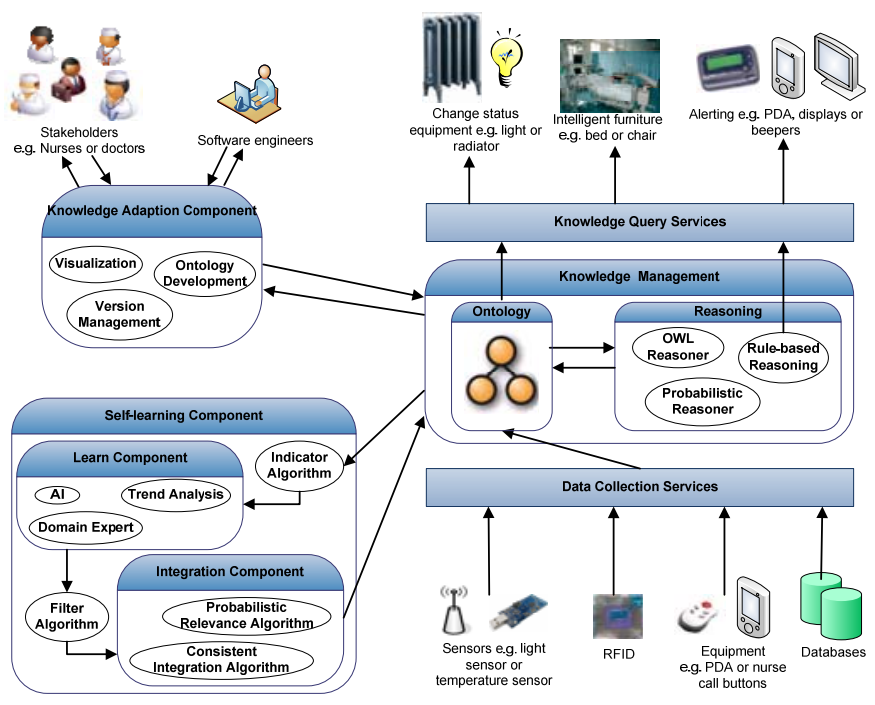

Figure 1. Architecture of the context-aware, ambient-intelligent framework

to implement the continuous care algorithms. The construction of the Ontology, using the Knowledge Adaption Component, is further detailed in Section III, while the implementation of the Rules is described in Section IV.

The Data Collection Services transform the inputted information to data that can be inserted in the ontology. For example, the Location Service is used by various devices to insert new information about the location of the patients and staff members into the Ontology. This new information can come from a Database or directly from a Device such as temperature and light sensors, RFID tags, PDAs and nurse call buttons. The Rules and OWL Reasoner are then used to reason on this information and derive certain conclusions. The Knowledge Query Services are used to transform this inferred data and conclusions to information that can be processed by the various Devices. This can result in changed status of a Device, such as dimming a light, or in a message that alerts a staff member display on for example a PDA.

As mentioned previously, the framework contains a Selflearning Component that makes the framework more adaptable to future needs. It observes the decisions and outcomes of the Rules and combines them with the information available in the Ontology to discover trends in the behavior of caregivers, nurses and patients. This information is then added to the Ontology or Rules to optimize them for continuous care. This component is further detailed in Section $\mathrm{V}$.

\section{ONTOLOGY DEVELOPMENT}

To facilitate the integration, analysis, combination and efficient usage of the context-aware data, an ontology is constructed that contains all the knowledge about the continuous care domain such as staff and patient profiles, environment and localization information and medical data. Although ontologies are widely accepted and adopted within the eHealth domain, most of these models focus on biomedical research such as the Gene Ontology [10] and National Cancer Institute ontology [11]. Little work has been done on the development of ontologies to support the continuous care of patients in a formal way. 
As mentioned previously, the development of this ontology is user-driven as this facilitates the acceptance of the new technology and empowers the staff members to take control of this ontology and adapt it to their needs. A lot of ontology engineering methodologies have been proposed in literature. The existing approaches are however rather extreme in their choices to include domain experts in the life-cycle of an ontology. On the one hand, there are methodologies [12] that only discuss the scope, the use and the requirements of the ontology with the domain experts during the specification space. The rest of the ontology life-cycle is completely controlled by the knowledge engineer. On the other hand, in the HCOME approach [9] the ontology is completely constructed by the domain experts by providing them with user-friendly and collaborative tools. For our framework, a methodology is used that finds a middle ground between these two extremes. The users are involved in each step of the life cycle of the ontology without having to construct the ontology themselves or attribute a large amount of their time.

Observations about the current daily practices and analysis of logging data from the available systems are used as starting point for the development of the ontology. A stakeholder group is created who are directly involved in the definition of the scope and the requirements of the ontology and the construction of scenarios about current practices. After finalization of this first stage, the knowledge engineers and stakeholders iteratively discuss the ontology under development. First a high-level ontology is created and represented to the stakeholders in a way that is concrete enough to evaluate it such as graph visualization, limited black box testing, wizard of ozz approach [13] or making tea approach [14]. The point of this procedure is not to overburden the stakeholders, since ontology development is not their primary interest, but giving care is. The ontology is further developed by taking the feedback of these stakeholders into account.

To prepare the ontology for the new ambient awareness it needs to support, participatory design sessions are organized with the stakeholders to reflect on these future care practices. Aim of these sessions is to stimulate the end users into creating scenarios/prototypes which represent how they want the ambient continues care process to be organized in the future and to reflect on the opportunities and bottlenecks of the changes in their current practices and context that could occur. Methods probes [15], mock-ups and proxies [16] are introduced to inspire and challenge the participants by making for example $3 \mathrm{D}$ sketches of the patient room of the future. The devised concepts during these sessions are included in the ontology to prepare it for future technologies.

Knowledge about a certain domain constantly changes such as the discovery of new drugs and diseases. An ontology is therefore not a static model, but a dynamic one that constantly should be able to change and evolve. This is taken into account during the construction of the ontology. The ontology is developed in such a way that new concepts, relations and definitions can easily be inserted into the ontology e.g. by defining very general concepts that can easily be divided in different sub-concepts. For example, one could define the measurements made about the condition of the patient e.g. Temperature, as individual concepts or as sub-concepts of a concept Measurement. Relations could then be defined on this general concept Measurement. When new measurements need to be added to the ontology e.g. Blood Pressure, it can easily be added as a sub-concept of Measurement. In this case it will inherit all the relations defined on Measurement automatically.

The ontology can be adapted in two ways. On the one hand, the Knowledge Adaption Component supports continuous development by providing visualization, ontology engineering tools and collaboration tools to the medical staff. This way the staff can easily adapt the ontology to future needs. On the other hand, the self-learning component automatically extends the ontology with new knowledge. This is further detailed in Section V.

\section{Rule-BASED AlgorithMS}

On top of this ontology intelligent Rule-based systems are developed that contain the reasoning algorithms to optimize and automate continuous care. These systems infer new knowledge (data) based on the data available in the ontology. This way they can readily adapt to the situation at hand. Efficient and fast notifications made by these systems allow appropriate actions to be taken in the continuous care setting.

An example of a situation that can be optimized this way is the assignment of a single nurse to multiple wards during the night shifts. At present, giving the nurse all the beepers of the wards for which he or she is responsible that night solves this situation. It often happens that a nurse is busy with one patient and a more urgent call comes in from a patient in another ward. The nurse then has to manually call another nurse to handle this urgent call. By employing a Rule-based algorithm on top of an ontology this situation can be handled more efficiently. The ontology keeps track of the current task of the nurses, their locations, the nature of the call (e.g. condition of the patient) and so on. A more intelligent rule-based algorithm runs on top of this ontology that takes all this context information into account. It reasons on the information in the ontology and decides not to call the responsible but busy nurse and call a less busy colleague that is sufficiently close to the patient to respond.

The developed algorithms (Rules) are directly derived from the use cases that are filtered out of the scenarios, which were identified during the first phase of the ontology development. This delivers general Rules such as "Only a nurse close enough to a patient can be called for a call made by this patient". Participatory design sessions between knowledge engineers and stakeholders are used to make this Rule more concrete by determining the parameters of this Rule. For instance, what is “close enough"? Iteratively testing, evaluating and discussing these Rules and their characteristics in the co-creation sessions leads to fully determined and implemented Rules. The Knowledge Adaption Component can also be used by staff members to adapt the Rules to fit their needs.

\section{SELF-LEARNING SYSTEM}

When new technology is introduced into an environment, the behavior of the actors changes to adapt to this new technology. This means that use cases on which the Rules are 
based may no longer apply. It is however difficult to predict which additional knowledge will be used. To make the system more adaptable to future needs, a self-learning component is being developed. It contains several algorithms to implement this functionality.

Firstly, a set of Indicator Algorithms determine missing knowledge in the ontology. An example of such an algorithm: a suggestion is given by the Rules to the caregivers to do a certain action, but the caregivers consequently do a different action under certain circumstances. These situations will be logged, so that they can be studied more closely.

Secondly, algorithms are provided by the Learn Component that can be used to analyze the logged situations, for example Trend Analysis and Artificial Intelligence (AI). These algorithms observe the decisions and outcomes of the Rulebased system and combine them with the information available in the ontology to discover trends in the behavior of caregivers, nurses and patients. A domain expert can also choose to study these situations him/herself.

Thirdly, the conclusions of Learn Component are studied further by the Filtering algorithms. These algorithms filter out of these conclusions the knowledge that should be added to the Ontology and the new Rules that should be defined. For example, if the AI outputs the data organized in clusters, these algorithms analyze the similarities in the data in these clusters and define new Rules.

Finally, the new knowledge can be integrated in the Ontology and the Rules with the Integration Component. The Consistent Integration Algorithms define probabilistic relations that connect this new knowledge to the existing knowledge. This way, it is made clear to the users that the knowledge is new and has not been confirmed by rigorous evaluation yet. Additionally, this probability makes sure that this new knowledge does not make the entire ontology inconsistent. The domain experts who determine the relevance of this new knowledge can assign this initial probability. However, Probabilistic Relevance Algorithms are also provided which automatically determine the initial probability of this knowledge. Additionally, these algorithms increase or decrease the probability depending on the new information that comes available about usage of this knowledge. Knowledge with a too small probability is eliminated from the ontology and knowledge with a very high probability is added to the ontology as generally accepted knowledge.

\section{CONCLUSION}

In this paper, a framework is proposed which combines the newest insights and developments in ICT to support the organization of continuous care provision. The framework employs an ontology to improve the integration and orchestration of all the involved technologies such as location ware services, nurse call systems, context-aware services and decision support systems. Rules are defined which take advantage of the information in the ontology to automate, optimize and personalize the continuous care of patients.
Stakeholders are involved in each step of the development cycle of the ontology and the accompanying algorithms to increase the adoption rate of this new technology and empower the users. The framework also provides a self-learning component, to make it more adaptable to future needs. Future work will mainly focus on further development and evaluation of this framework on some specific use cases.

\section{ACKNOWLEDGMENT}

We would like to acknowledge that part of this research was supported by the IBBT Project ACCIO co-funded by the IWT, the IBBT and the following partners: Televic NV, Boone NV, Dominiek Savio Instituut and In-Ham. Femke Ongenae would like to thank the IWT for financial support through her Ph.D. grant.

\section{REFERENCES}

[1] M. Tentori, D. Segura and J. Favela, "Monitoring hospital patients using ambient displays," in Mobile Health Solutions for Biomedical Applications, P. Olla and J. Tan, Eds. USA: Medical Information Science Reference, 2009, Chapter VIII, pp. 143-158.

[2] J.-C. Burgelman and Y. Punie, "Close encounters of a different kind: ambient intelligence in Europe," in True Vision: The Emergence of Ambient Intelligence, E. H. L. Aarts and J. L. Encarnaçeo, Eds. Berlin: Springer Verlag, 2006, pp. 19-35.

[3] Y. Punie, "The future of ambient intelligence in Europe: the need for more everyday life," Communication and Strategies, Nr. 57, pp. 141165, Jan. 2005.

[4] J. Criel and L. Claeys, "A transdisciplinary study design on context aware applications and environments. A critical view on user parcticipation within calm computing," Observatio), Vol. 2, Nr. 2, 2008.

[5] A. Rullo, "The soft qualities of interaction," ACM Trans. Comput.-Hum. Interact., Vol. 15, Nr. 4, Nov. 2008.

[6] T. R. Gruber, "A translation approach to portable ontologies", Knowledge Acquisition, Vol. 5, Nr. 2, pp. 199-220, 1993.

[7] S. Bechhofer, F. Van Harmelen, J. Hendler, I. Horrocks, D. L. McGuinness, P. F. Patel-Schneider, L. A. Stein, "OWL Ontology Web Language - Reference”, W3C Recommendation 2004.

[8] J. J. Caroll, I. Dickinson, C. Dollin, D. Reynolds, A. Seaborne, K. Wilkinson, "Jena: Implementing the semantic web recommendations," In Proc. of the $13^{\text {th }}$ Int. World Wide Web Conference, New York, USA, 2004, pp. 74-83.

[9] K. Kotis and G. A. Vouros, "Human-centered ontology engineering: The HCOME methodology," International Journal of Knowledge and Information Systems (KAIS), Vol. 10, Nr. 1, pp 109-131, 2006.

[10] The Gene Ontology: http://www.geneontology.org/

[11] The NCI Cancer Ontology: http://ncicb.nci.nih.gov/

[12] H. S. Pinto and J.P. Martins, "Ontologies: How can they be built?" Journal of Knowledge and Information Systems, Vol. 6, Nr. 4, pp. 441464, 2004.

[13] L. Molin, "Wizard-of-Oz prototyping for co-operative interaction design of graphical user interfaces," In Proc. of the $3^{\text {rd }}$ Nordic Conference on Human-Computer Interaction, October 23 - 27, 2004, pp. 425-428.

[14] A. Smith and L. Dunckley, "Prototype evaluation and redesign: structuring the design space through contextual techniques," Interacting with Computers, Vol. 14, Nr. 6, pp. 821-843, 2002.

[15] K. Boehner, J. Vertesi, P. Sengers and P. Dourish, "How HCI interprets the probes", In Proc. of the SIGCHI Conference on Human Factors in Computing Systems, April 28 - May 03, 2007, pp. 1077-1086.

[16] J. Preece, Y. Rogers, and H. Sharp, Interaction Design: Beyond HumanComputer Interaction, UK: Wiley \& Sons, 2002. 